
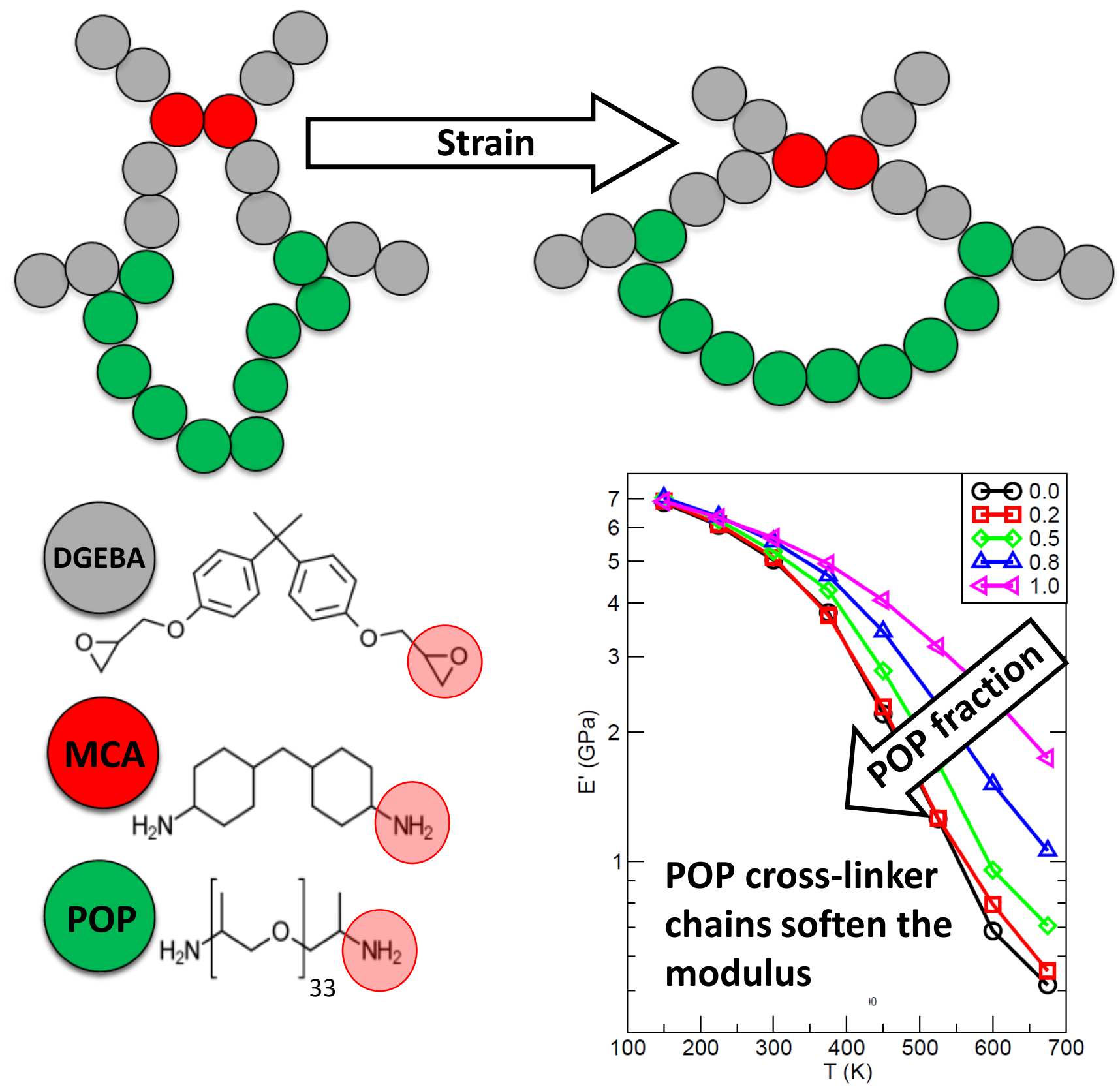


\title{
Bi-modal Polymer Networks: Viscoelasticity and Mechanics from Molecular Dynamics Simulation
}

\author{
Timothy W. Sirk ${ }^{\mathrm{a}}$, Mir Karim ${ }^{\mathrm{b}}$, Joseph L. Lenhart ${ }^{\mathrm{a}}$, Jan W. Andzelm ${ }^{\mathrm{a}}$, Rajesh Khare ${ }^{\mathrm{b}}$ \\ ${ }^{a}$ Macromolecular Science and Technology Branch, U.S. Army Research Laboratory, Aberdeen Proving Ground, Maryland, \\ United States. E-mail: tim.sirk@us.army.mil \\ ${ }^{b}$ Department of Chemical Engineering, Texas Tech University, Box 43121, Lubbock, Texas 79409, United States. E-mail: \\ rajesh.khare@ttu.edu
}

\begin{abstract}
The high strain-rate rheological and mechanical properties of bi-modal epoxy polymer networks were characterized using molecular dynamics simulation. The complex Young's modulus was found by applying a cyclic sinusoidal strain over a wide range of temperatures spanning the glass transition. The non-linear stress response was studied in the glass transition region using uni-axial deformation. We discuss special considerations in computing viscoelastic properties at the high strain-rates available to molecular dynamics. As in experimental studies, the complex modulus is shown to be a function of the network composition and strain rate. However, the high strain-rate simulations performed here predict the existence of broad peaks in the temperature-dependent loss modulus and slow relaxation of the storage modulus. In general, it is observed that network compositions with larger amounts of short, stiff 4,4'methylenebis(cyclohexylamine) (MCA) cross-linkers lead to an increase in the mechanical glass transition temperature as well as the breadth of the glass transition compared to longer, more flexible poly(oxypropylene) diamine (POP) cross-linkers. When the networks of any composition were deformed beyond the linear region, the stress response displayed a plateau that was associated with the extension of network chains.
\end{abstract}

(C) 2011 Published by Elsevier Ltd.

Keywords: molecular dynamics, mixed network, viscoelasticity, epoxy, high strain-rate

\section{Introduction}

Thermoset polymer networks comprise a diverse and widely-useful category of protective and structural materials found in both civilian and military applications. Laboratory experiments of ballistic penetration have identified key factors controlling high-rate impact performance of epoxy networks. While the nonequilibrium state of such experiments is complex, with time- and spatially-dependent distributions of stress, 
strain and strain-rate, the ballistic performance has been correlated with linear mechanical properties[1] and thermal transitions such as the glass transition[2].

Recent experiments by Masser et al. have shown that networks composed of diglycidyl ether of bisphenol A (DGEBA) monomers reacted with mixtures of stiff 4,4'-methylenebis(cyclohexylamine) (MCA) and flexible poly(oxypropylene) diamine (POP) cross-linkers are capable of tremendous improvements of ballistic performance compared with the neat epoxy networks. [3] In that work, the addition of a rigid MCA crosslinker served to broaden the temperature-dependence of the modulus such that it did not vary substantially over operational temperatures. Furthermore, flexible spacer units were incorporated as POP cross-linkers, which discouraged the brittle failure typically found in rigid networks. The mixture of rigid and flexible cross-linkers did not phase separate on the macro-scale, however two glass transitions were observed with dielectric spectroscopy. [3]

We postulate that materials which exhibit broadened or multiple glass transitions can exhibit improved ballistic performance over a broad temperature range due to the increased probability that an impact event will interact strongly with the glass transition. Here, we use molecular dynamics simulations to investigate in detail the complex modulus and non-linear mechanics of such bi-modal epoxy networks. We consider representative models of POP:MCA:DGEBA compositions. The high strain rate rheology and nonlinear mechanics of these compositions are studied by applying oscillatory strains and uni-axial deformation, respectively. The length- and time-scales available with molecular dynamics simulations are well-suited for this task, since the dynamics of fully-resolved (atomistic) thermoset structures can be studied at strain rates much higher than those typically available to experiments. This paper is organized as follows: In Section 2, details of the molecular dynamics simulations are described, including the forcefield, description of the polymer network, and the annealing procedure. Section 3 discusses considerations in simulating high strain-rate rheological properties; Section 4 presents the results of mechanical tests; Section 5 summarizes the broader meaning of our results.

\section{Simulation Details}

We use epoxy network structures from a previous study[4]; the methods of construction and equilibration procedure of these epoxy networks are described in detail elsewhere [5, 4]. In short, atomistic models of fully reacted epoxy network were created using a simulated annealing polymerization technique applied to polymer networks[6]. Stoichiometric mixtures were considered for DGEBA monomers with MCA and POP cross-linkers (see Figure 1), where the relative amounts of the two cross-linkers were changed to create systems with different compositions. We use the parameter $x$ to represent the mole fraction of MCA in the cross-linker mixture. In this study, five network compositions were used $(x=0.0,0.2,0.5,0.8,1.0)$ with each model system containing approximately 200,000 atoms. The POP and MCA cross-linkers and DGEBA monomers were well-mixed before curing; after curing, we found that most cross-linkers $(86 \%)$ shared at least one monomer with a different type of cross-linker if the cross-linker fractions were equal $(x=0.5)$. Loop defects were prevented during curing by disallowing any pair of monomer and cross-linker molecules from sharing more than one cross-link bond. For each composition $x$, we use five independent replica structures, where systems are selected in the temperature range of 150-675 $\mathrm{K}$ and in temperature increments of 75 $\mathrm{K}$. The GAFF force field $[7,8]$ was used with tail corrections [9] for computing energy and pressure of the Lennard-Jones potential. Partial atomic charges were computed with the AM1-BCC method[10, 11] and the particle-particle particle-mesh (PPPM) method[12] was used for electrostatic interactions. All of the simulations were carried out using the LAMMPS package[13] with a timestep of 1.0 fs using Nosè-Hoover control of temperature and pressure implemented according to the equations of motion given by Shinoda[14]. Results were averaged over the replica structures and, for cyclic deformations, over 100 strain cycles per replica. 

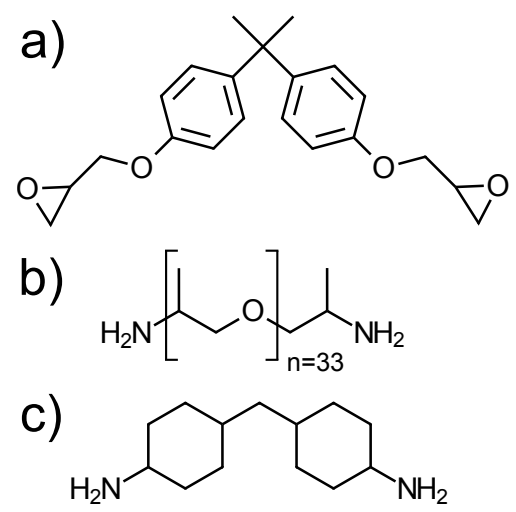

Figure 1. Chemical structures of (a) DGEBA (b) POP and (c) MCA.

Table 1. Details of the epoxy network systems. The mole fraction of MCA cross-linkers $(x)$ and the corresponding number of molecules $(N)$ and number of atoms $(n)$ are shown for each of the mixed networks.

\begin{tabular}{ccccc}
\hline$x$ & $N$-POP & $N$-MCA & $N$-DGEBA & $n$ \\
\hline 0.0 & 450 & 0 & 900 & 199350 \\
0.2 & 420 & 105 & 1050 & 200655 \\
0.5 & 343 & 343 & 1372 & 199626 \\
0.8 & 200 & 800 & 2000 & 199800 \\
1.0 & 0 & 1360 & 2720 & 189040 \\
\hline
\end{tabular}

\section{Rheology at High Strain Rates}

\subsection{Background}

Among the mechanical properties, Young's modulus is the most commonly measured in the simulation literature. In a typical calculation of Young's modulus using dynamics, a small uni-axial strain $(\epsilon)$ is imposed (usually $1 \%-3 \%$ ) at a strain rate of $10^{6}-10^{10} \mathrm{~s}^{-1}$, and the resulting axial stress $(\sigma)$ response is used to determine the Young's modulus as $E=\Delta \sigma / \Delta \epsilon$. This approach is most effective well-below $T_{g}$, where the storage modulus is high, the stress response is large, and the uncertainty of stress measurements is low. At temperatures approaching and exceeding $T_{g}$, the stress response can become comparable to the stress fluctuations. We have recently shown that this problem can be alleviated in tightly crosslinked networks by using large model structures of $\sim 200,000$ atoms; it was shown that the rubbery behavior of the Young's modulus can be observed by applying a 1\% uni-axial deformation and averaging over six replica.[15] In this work, we strive to better characterize the mechanical glass transition at high rates by decomposing the moduli into viscoelastic storage and loss modulus. Linear viscoelastic properties can be determined from molecular simulations by several methods, such as applying a step-strain or step-stress followed by a relaxation process[16] or applying oscillatory strain or stress. Here, we find the complex Young's modulus by applying a small, sinusoidal strain of $0.1 \%$ amplitude. Such a strain can be imposed by altering the edge length $L$ of the simulation box as

$$
L(t)=L(0)+L_{0} \sin (\omega t)
$$

where $L_{0}$ and $\omega$ are the amplitude and frequency of the length oscillation. The time-dependent stress response is taken as

$$
\sigma(t)=\sigma(0)+\sigma_{0} \sin (\omega t+\delta)
$$

where $\sigma(0)$ is the undeformed stress, $\sigma_{0}$ is the stress amplitude, and $\delta$ is the phase shift from $L(t)$. Eq. 2 is often restated as 


$$
\sigma(t)=\sigma(0)+\epsilon_{0}\left[E^{\prime}(\omega) \sin (\omega t)+E^{\prime \prime}(\omega) \cos (\omega t)\right],
$$

where the storage $\left(E^{\prime}\right)$ and loss modulus $\left(E^{\prime \prime}\right)$ represent the degree of elastic energy storage and viscous dissipation, respectively, and $\epsilon_{0}=L_{0} / L(0)$. From the literature, cyclic deformations of atomistic models have been carried out for only a small number of polymers[17, 18, 19] and polymer networks[20] for the purpose of studying yield and hysteresis.

\subsection{Upper Limits of Frequency}

The timescales available to classical molecular dynamics simulation are well-suited to study high strain rate mechanical behavior. In what follows, we discuss several considerations in establishing a steady-state response from an oscillatory strain of frequency $\omega$ (with period $\tau_{p}$ ). Due to the large distance between crosslinks, the composition $x=0.0$ is expected to have the lowest modulus and therefore require the highest strain frequencies to study the glass transition. Unless stated otherwise, we consider only the composition $x=0.0$ to determine the upper limits of the frequency.

Isothermal Condition. The steady-state deformation of a viscoelastic material is expected to produce internal heating. For an isothermal deformation to occur, thermal conduction must at least allow the internally-generated heat current to transport across the simulation box of length $L$ on a timescale $\tau_{d}$ that is much smaller than the period of an oscillation, i.e., $\tau_{d} / \tau_{p}<<1$. Taking $\tau_{d}=L^{2} / \alpha_{T}$, where $\alpha_{T}$ is the thermal diffusivity,

$$
\frac{\omega L^{2}}{\alpha_{T}}<<1 .
$$

For our box of $L \approx 13 \mathrm{~nm}$, estimating $\alpha_{T}=10^{-3} \mathrm{~cm}^{2} / \mathrm{s}$ for crosslinked epoxy[4], and requiring $\tau_{d} / \tau_{p}=0.1$ leads to a maximum frequency of $\omega \approx 10^{9} \mathrm{~Hz}$ to achieve a steady-state, isothermal behavior in the crosslinked epoxy system studied here. However, the expected increase in temperature over a single cycle is not necessarily important. The energy accumulated per cycle, $\Delta \Phi$, can be found by integrating the stress over a strain cycle as

$$
\Delta \Phi=\int_{-\varepsilon_{0}}^{\varepsilon_{0}} \sigma d \varepsilon=\int_{0}^{2 \pi / \omega} \sigma \dot{\varepsilon}(t) d t
$$

or equivalently $\Delta \Phi=\pi \varepsilon_{0}^{2} E^{\prime \prime}$. The temperature increase per unit volume can be estimated over a single cycle from an energy balance as $\Delta \Phi=c_{p} \Delta T / v$. For the network composition $x=0.0$ at $435 \mathrm{~K}$, we have previously[4] found the heat capacity and specific volume of this system as $c_{p}(435 K) \approx 4.7 \mathrm{~J} / \mathrm{g} / \mathrm{K}$ and $v \approx 0.98 \mathrm{~cm}^{3} / \mathrm{g}$, respectively. Assuming a loss modulus of $E^{\prime \prime}=1.0 \mathrm{GPa}$, our model predicts only a small increase in temperature $(\Delta T<0.1 \mathrm{~K})$ per cycle. We therefore choose to perform isothermal, rather than adiabatic, deformations with the understanding that each cycle represents an independent test, i.e., the accumulated temperature increases are not considered. This assumption is useful with cyclic deformations since, in the absence of stress ratcheting[20] or fatigue, the deformation can be repeated any number of cycles to improve the quality of stress data.

Elastic Response. Cyclic stress-strain plots, known as Lissajous-Bowditch (L-B) curves, are often used in the study of elastoviscoplastic materials. Constitutive laws can be recognized in L-B curves by several characteristic shapes and their intermediate forms, such as circular (viscous), linear (elastic), and rectangular (perfectly plastic). The energy dissipation evaluated from Eq. 5 has an upper bound set by $4 \sigma_{0} \epsilon_{0}$ for a purely plastic response, and a lower bound of zero set by an elastic response. We require the energy dissipated in the glassy state to be small compared with a plastic response, i.e.,

$$
\frac{\Delta \Phi}{4 \sigma_{0} \varepsilon_{0}}<<1
$$

A metric similar to Eq. 6 has been used to study the yield of elastoviscoplastic materials during large amplitude oscillatory stain.[21] To test Eq. 6, oscillatory strain for four different frequencies $\left(\omega=5 \cdot 10^{9}, 5\right.$. 


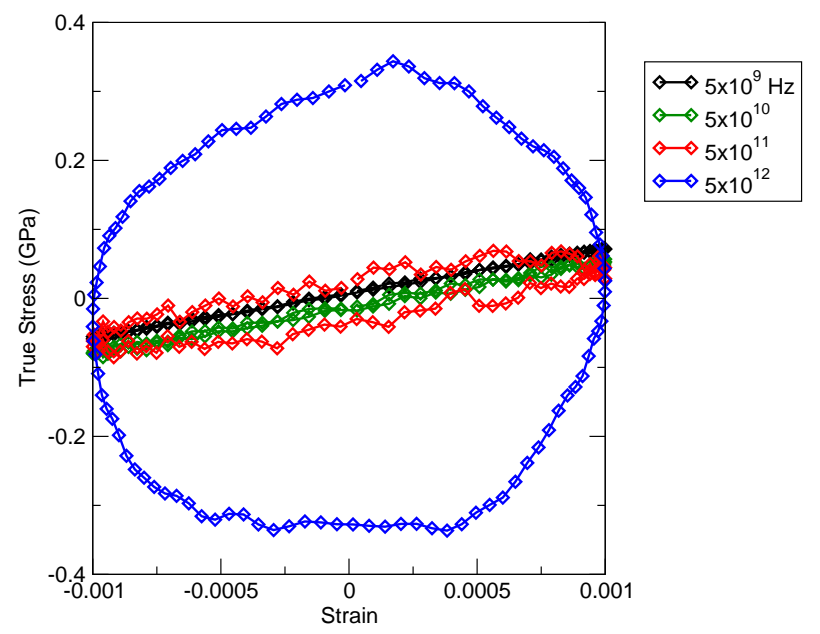

Figure 2. Lissajous-Bowditch plot for the composition $x=0.0$ in the glassy state $(150 \mathrm{~K})$.

$10^{10}, 5 \cdot 10^{11}$ and $5 \cdot 10^{12}$ ) was applied to the network mixture of $x=0.0$. The results are shown in Figure 2 as an L-B curve. As shown in the figure, the two highest frequencies resulted in strongly inelastic deformations. In particular, it is clear that a fundamental change occurs at the highest frequency $\left(5 \cdot 10^{12} \mathrm{~Hz}\right)$ in which the stress curve begins to track the strain rate rather than the strain, i.e., the highest stresses occur at low strains (high strain rate) and the lowest stresses at larger strains (low strain rate). To quantify the loss, $\Delta \Phi / 4 \sigma_{0} \varepsilon_{0}$ was evaluated at each frequency by finding $\Delta \Phi$ (from Eq. 5) and the plastic stress response $4 \sigma_{0} \varepsilon_{0}$. As shown in Figure 3, the resulting values decreased sharply at $5 \cdot 10^{10} \mathrm{~Hz}$, thus it is clear that the response at higher frequencies is strongly inelastic. To better understand this result, we consider the characteristic frequency of non-bonded atomic collisions, which we previously estimated to be on the order of $10^{13} \mathrm{~Hz}$.[4] Because the highest mechanical frequencies considered in Figure 2 (e.g., $5 \cdot 10^{12} \mathrm{~Hz}$ ) are of the same order, we expect local relaxations to be reduced or frozen during the strain cycle and, as a result, unphysical atomic collisions to be created when the atomic coordinates are mapped to a new value of strain.

Strain Rate. The propagation of stress waves can become important in boundary-driven deformation at high strain rates. For a small sinusoidal deformation, $L(t) \approx L, v(t)=\epsilon_{0} L \omega \cos (\omega t)$, and the speed of sound $c$ is approximately constant. We choose to neglect wave propagation effects when a stress wave travels through the box on a timescale $\tau_{w}$ that is much faster than the period of oscillation, i.e., $\tau_{w} / \tau_{p}<<1$. The characteristic time for a stress wave of speed $c$ to cross the simulation box is taken as $\tau_{w}=L /(c-v(t))$, where $\tau_{w}$ is maximized when $v(t)=\epsilon_{0} L \omega$. Therefore, we require

$$
\frac{\omega L}{c-\epsilon_{0} L \omega}<<1 .
$$

If Eq. 7 is not satisfied, the stress response can be expected to depend on both time and position, as is the case with shock simulations[22]. Otherwise, a choice can be made in the deformation protocol. The wave propagation can be preserved by deforming the system through a boundary-driven oscillation, or neglected by enforcing an affine transformation of the atomic positions. The boundary-driven oscillation does not have a clear physical interpretation under periodic boundary conditions, thus we apply the affine transformation. The upper frequency limit corresponding to the affine assumption can be estimated from Eq. 7 by taking the wave speed as $c=\sqrt{K v}$, where $K$ is the bulk modulus. To determine the bulk modulus, we find the components of the stiffness matrix $C_{i j}$ and apply $K=\left(C_{11}+C_{12}+C_{21}+C_{22}\right) / 4$. The stiffness matrix can be defined in Voigt notation as

$$
C_{i j}=\frac{1}{V}\left[\frac{\partial^{2} A}{\partial \epsilon_{i} \partial \epsilon_{j}}\right]_{T}=\frac{1}{V}\left[\frac{\partial^{2} U}{\partial \epsilon_{i} \partial \epsilon_{j}}\right]_{T}-\frac{T}{V}\left[\frac{\partial^{2} S}{\partial \epsilon_{i} \partial \epsilon_{j}}\right]_{T}
$$




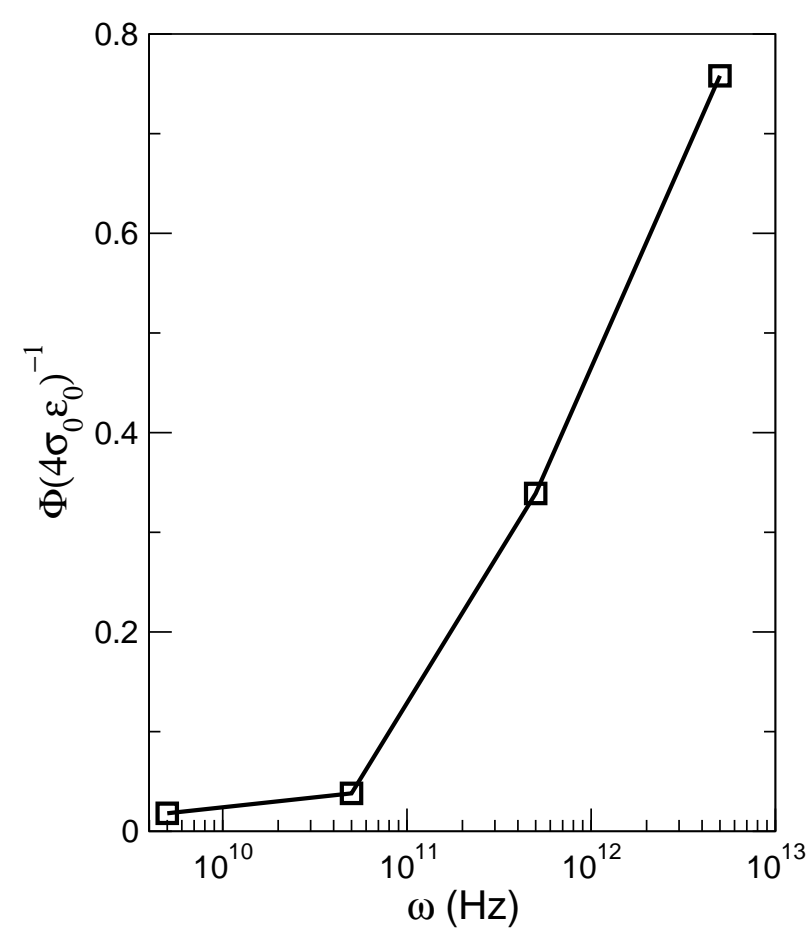

Figure 3. Fraction of energy dissipation $\left(\Delta \Phi / 4 \sigma_{0} \varepsilon_{0}\right)$ observed in the glassy state as a function of frequency $(\omega)$ for the composition $x=0.0$.

where $A, U$ and $S$ are the Hemholtz free energy, internal energy, and entropy, respectively. For the purpose of estimating the bulk modulus, we apply the "static method", i.e, a strain is imposed in the $i$ th and $j$ th directions, energy minimization is performed, and $C_{i j}$ is taken from the gradient of the resulting stress tensor. The static method is well-known to neglect the entropic contributions corresponding to the last term of Eq. 8, and it is established that elastic constants of amorphous polymers computed with the static method are typically greater than those from dynamics, presumably due to the neglect of entropy. Nonetheless, the static method remains an efficient method for estimating mechanical constants deep in the glassy state. The error due to neglect of the entropic contribution was previously taken from thermodynamic considerations [23, 24] and the deviation from linear elasticity during dynamics[25]. Here, we estimate the entropic contribution using an approach similar to that of In't Veld et al.[25], in which the stress response during dynamics is decomposed into energetic and entropic terms by performing a Taylor series expansion of stress around a strain value,

$$
\sigma(\epsilon)=\sigma\left(\epsilon_{0}\right)+\left.\epsilon \frac{\partial \sigma}{\partial \epsilon}\right|_{0}+\left.\frac{\epsilon^{2}}{2} \frac{\partial^{2} \sigma}{\partial^{2} \epsilon}\right|_{0}+\left.\ldots \frac{\epsilon^{n}}{n !} \frac{\partial^{n} \sigma}{\partial^{n} \epsilon}\right|_{0} .
$$

The second term of Eq. 9 represents the stress contributions from linear elasticity, and the higher order terms represent the stress contribution from all other sources, including the entropic term. To understand the importance of the entropic contribution in the glassy state during dynamics, we computed the Taylor series during a series of deformations. Eq. 9 was evaluated by considering the stresses at ten small strains centered around $\epsilon_{0}$ (taken within a range of $\epsilon_{0}+/-0.033 \%$ strain) and truncating the series at $n=5$. To improve the sampling, we repeated this process for ten values of $\epsilon_{0}$ near zero strain. The ratio of the higher order terms to the second term was about $19 \%$. We consider this error as acceptable for the current purpose of establishing frequency limits, and apply the static method to estimate the bulk modulus in the glassy state.

The stiffness matrix was computed by applying a set of small strains to five replica structures of one 
network composition $(x=0.0)$, followed by energy minimization using the conjugate gradient method (e.g., see Theodorou and Suter[23]). To ensure that $C_{i j}$ is independent of strain, six strain values were attempted for the network composition $x=0.0: \epsilon=0.001 \%, 0.01 \%, 0.05 \%, 0.1 \%, 0.5 \%$, and $1 \%$; The resulting values of bulk modulus were $K=41.6,10.67,8.22,8.12,7.96,7.71 \mathrm{GPa}$, respectively. We take $K=8.12 \mathrm{GPa}$, and compute the speed of sound as $c=2645 \mathrm{~m} / \mathrm{s}$. The standard of satisfying the inequality in Eq. 7 should not be excessively conservative, as this value is closely related to computational effort. For $\tau_{w} / \tau_{p}=0.1$ and a typical system size of $L \approx 13 \mathrm{~nm}$, the maximum estimated frequency is $\omega=1.84 \cdot 10^{11} \mathrm{~Hz}$. A similar analysis considering a network composition of $x=1.0$ leads to an estimate of $K=8.8 \mathrm{GPa}, c=2774 \mathrm{~m} / \mathrm{s}$, and $\omega=1.94 \cdot 10^{11} \mathrm{~Hz}$. If the $K$ of the polymer networks is reduced by a factor of four above the glass transition, the maximum frequency can be further limited as $\sqrt{1 / 4} \omega=9.7 \cdot 10^{10} \mathrm{~Hz}$.

Inertial Forces. As a final check, we compare the stress resulting from changes in momentum with the viscoelastic stress. The maximum acceleration in our sinusoid strain $\left(\epsilon_{0} L \omega^{2}\right)$ occurs at zero strain rate. We take the stress from momentum change as $\sigma=(L / v) \frac{d^{2} x}{d t^{2}}=\epsilon_{0} L^{2} \omega^{2} / v$. Because the strain rate is zero at the maximum acceleration, we neglect the strain-rate-dependent viscous stress and consider only the straindependent elastic stress as $\sigma=E^{\prime} \epsilon_{0}$. Requiring the elastic stress to be much larger than the momentum stress leads to

$$
\frac{\omega^{2} L^{2} / v}{E^{\prime}}<<1 .
$$

By requiring the LHS of Eq. 10 as 0.1 and choosing properties at $150 \mathrm{~K}\left(v=0.875 \mathrm{~cm}^{3} / \mathrm{g}, E^{\prime} \approx E=7.0\right.$ $\mathrm{GPa}, L=12 \mathrm{~nm}$ ), the limiting frequency is predicted as $6.5 \cdot 10^{10} \mathrm{~Hz}$ for the glassy state. At very high temperatures, the modulus in the rubbery region can be expected to drastically reduce and approach the predictions of the theory of rubbery elasticity; in that case, Eq. 10 would require much lower frequencies. We choose a frequency of $5 \cdot 10^{10} \mathrm{~Hz}$ for the remaining simulations (unless otherwise stated) and neglect inertial effects by applying an affine deformation of the coordinates. To aid in comparison with tensile tests, an averaged linear strain rate can be found as the ratio of the strain amplitude and one-fourth of the period, which results in a value of $10^{8} \mathrm{~s}^{-1}$. We note that strain rates of this magnitude are commonly used in atomistic molecular dynamics simulations of glassy polymers (e.g., Ref. [26]).

\section{Mechanical Properties}

\subsection{Complex Modulus}

The complex Young's modulus was found by applying a sinusoidal strain according to Eq. 1. For each network composition, eight temperatures spanning the glass transition region were chosen between 150-675 $\mathrm{K}$ in increments of $75 \mathrm{~K}$, where the equilibrated structures were taken from our previous work [4]. The stress response at each temperature was fit to Eq. 3, and $E, E^{\prime}$ and $E^{\prime \prime}$ were found as $\sigma_{0} / \epsilon_{0}, E \cos (\delta)$, and $E \sin (\delta)$, respectively.

As a representative example, we consider the complex modulus of the $x=0.0$ system, shown in Figure 4. At the low temperatures corresponding to the glassy state, the Young's modulus $(E)$ is large, decreases slowly with temperature, and is dominated by the elastic contributions $\left(E^{\prime}\right)$. As temperature increases, $E$ decreases more quickly, the slope of $E^{\prime}$ steepens, and $E^{\prime \prime}$ increases until reaching a maximum. The abrupt drop in $E^{\prime}$ and maximum of $E^{\prime \prime}$ are associated with the mechanical glass transition. As temperature is increased further, $E^{\prime \prime}$ is reduced, and the slopes of $E$ and $E^{\prime}$ decrease.

The temperature-dependent modulus from dynamic modulus analysis (DMA) experiments of glassforming networks has the following general features: the glassy stress response at small strains is dominated by reversible elastic contributions (represented by $E^{\prime}$ ) which are considered to be due to the local displacement of tightly packed atoms or groups of atoms; in the glass transition region, coordinated motion across numerous chain segments occurs which results in viscous contributions to the stress (represented by $E^{\prime \prime}$ ) becoming comparable to elastic contributions; at temperatures well-above the glass transition, the stress is again dominated by elastic contributions due to stretching of the network chains. The simulation trends we report have many of the same features observed in experiments, therefore we focus our discussion on the 
differences. First, the modulus in our molecular simulations did not reach a rubbery plateau that is typical in DMA experiments, i.e., $E^{\prime}$ is still decreasing at our highest temperature of $735 \mathrm{~K}$. Although, the trend of $E^{\prime}$ suggests the beginning of a rubbery plateau modulus, we did not explore the very high temperatures required to reach the plateau. We also note that the maximum ratio of loss to storage modulus $(\tan \delta)$ is often reported in experimental studies; we do not report this value, as it also occurs beyond our highest temperature of $735 \mathrm{~K}$. Relative to experiments, the rubbery modulus and peak of $\tan \delta$ exist at elevated temperatures due to both the shift of the glass transition to a higher temperature, and the increase in the breadth of the glass transition.

Previously, we observed a gradual transition of Young's modulus across $T_{g}$ in simulations of uni-axial elongation at constant strain rates.[5] Similar behavior of the glass transition is seen in Figure 5. For each of the networks, the slope of $E^{\prime}$ in the glass transition region is more gradual than typical experimental results of DMA for epoxy[27], which are taken at a frequencies near $1 \mathrm{~Hz}$ and occur over a temperature range of 20-30 K. [27, 28] The increased breadth of glass transition with frequency has been attributed to the overlap of the $\beta$ - and $\alpha$-transitions.[5] The glass transition can also be expected to broaden due the temperature-dependence of the relaxation time. In a recent work, Schmelzer and Tropin [29] estimated the expected breadth of the glass transition by asserting that the timescale associated with the glass transition temperature must be increased or decreased by a multiplier factor in order to observe the glassy or rubbery states, respectively. The authors found that a small temperature change was sufficient to alter the timescale if the $T_{g}$ was low, however, a progressively larger temperature change was needed as the $T_{g}$ increased. In the case of the Vogel-Fulcher-Tammann (VFT) relaxation, the breadth of the glass transition was predicted as

$$
\delta T_{g}=\frac{C}{B^{2}}\left(T_{g}-T_{0}\right)^{2}
$$

where $C$ is a factor describing the ratio of the timescales needed to observe the glass transition, $B$ is a VFT parameter, and $T_{0}$ is the Vogel temperature. From Eq. 11, it is clear that the glass transition is expected to rapidly broaden as it moves above the Vogel temperature. If validity of the VFT equation is assumed across the entire temperature range, $\delta T_{g}$ can be estimated as follows. In low rate experiments, McAninch et al. [28] reported $\delta T_{g}$ and $T_{g}$ of the $x=0.0$ system as $20 \mathrm{~K}$ and $235 \mathrm{~K}$, respectively; substituting these values into Eq. 11 and assuming $T_{0}=T_{g}-50 \mathrm{~K}$, leads to $C \approx 0.008$. Using this value of $C, \delta T_{g}$ can now be estimated under high rate conditions that elevate the $T_{g}$. For $T_{g}=425 \mathrm{~K}$, the predicted $\delta T_{g}$ is approximately $450 \mathrm{~K}$, a value similar to the breadth of the loss modulus in Figure 4.

Changing the composition of crosslinkers greatly affected the breadth of glass transition region and value of the complex modulus near $T_{g}$. The temperature dependence of $E^{\prime}$ and $E^{\prime \prime}$ is shown for each network mixture in Figure 5. The value of the storage modulus for temperatures deep in the glassy region was about $7 \mathrm{GPa}$ for all of the networks and, similarly, the maximum of the loss modulus was near 1.0 GPa for all networks. Differences in the modulus are apparent near the glass transition, where the breadth of glass transition of $E^{\prime}$ was generally found to increase with $x$, i.e., systems with purely MCA cross-linkers have the broadest storage modulus. This trend is not observed in low strain-rate experiments, rather the experimental modulus of single-component networks have sharper transitions compared with network mixtures.[3] These experimental results have been interpreted as resulting from a broader relaxation spectrum available in network mixtures, which therefore relax over a broader range of temperatures. For the high-rate simulations here, we expect the width of the glass transition to depend on both the network composition, and on the increase of the $T_{g}$ from Eq. 11 (provided that the Vogel temperature $T_{0}$ is approximately constant). We have shown the $T_{g}$ of our compositions to increase strongly with MCA content[4], and we attribute the broad width of the glass transition for $x=1.0$ to its high $T_{g}$ and the form of Eq. 11 .

The position of the loss peak relative to the volumetric glass transition is very interesting (see Figure 6). We have previously reported epoxy simulations in which the $T_{g}$, as computed from volumetric and enthalpic changes during cooling, was well-above experimental values; this difference was understood to be due to the high cooling rates of molecular dynamics simulation which cause the system to fall out of equilibrium at an elevated temperature. $[5,4]$ Despite this upward shift of the $T_{g}$ relative to experiments, Figure 6 shows that the mechanical glass transition temperature, as measured by the peak of the loss modulus, is higher 


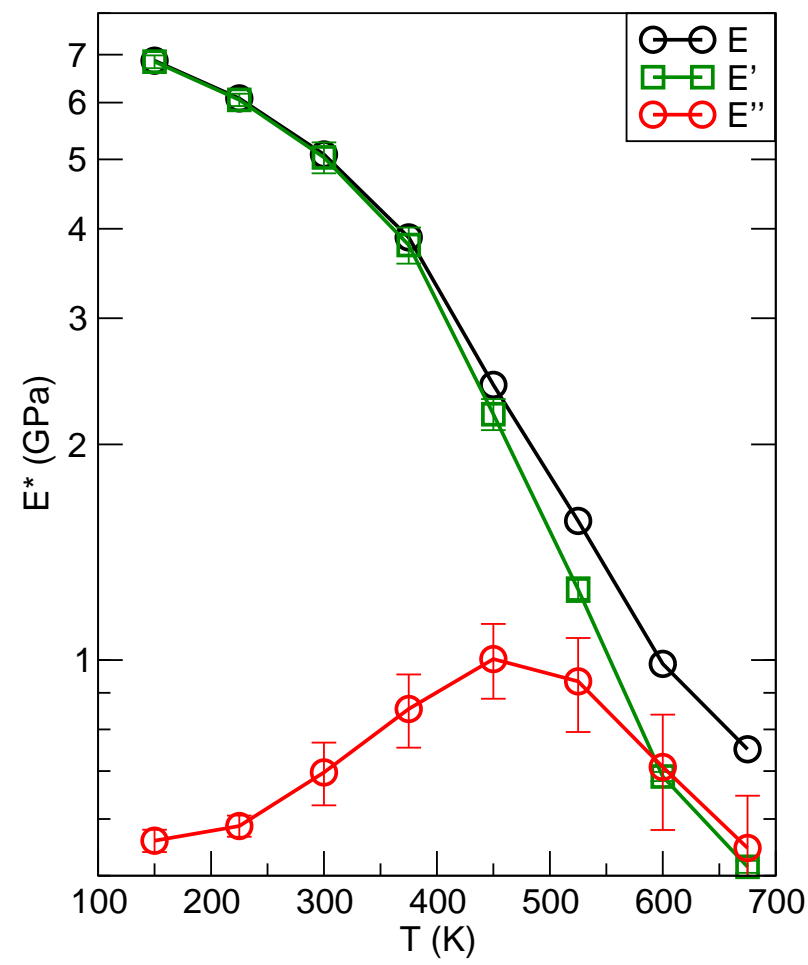

Figure 4. Complex Young's modulus of the composition $x=0.0$, as measured by applying a cyclic strain with a frequency of $5 \cdot 10^{10} \mathrm{~Hz}$. The total $(E)$, storage $\left(E^{\prime}\right)$, and loss $\left(E^{\prime \prime}\right)$ modulus are shown as a function of temperature $(T)$.

than the volumetric $T_{g}$ by approximately $100 \mathrm{~K}$ for the materials considered here. In this work, studying the glass transition of $E$ at temperatures above the volumetric $T_{g}$ was desirable, since the thermodynamic properties of the system are independent of the cooling rate under these conditions.

To rationalize the differences in the volumetric and mechanical $T_{g}$, we compare the timescale of the mechanical deformation, taken as $\tau_{m}=\dot{\epsilon}^{-1}$, with a timescale of cooling, taken as $\tau_{c}=\dot{\epsilon}_{c}^{-1}$ where $\epsilon_{c}$ is the strain along a dimension of the simulation cell during cooling. From our previous study of the same systems[4], the volume-temperature diagram indicates $\tau_{c}$ ranges between $\sim 10^{-6}-10^{-5} \mathrm{~s}$. This range of timescales is much slower than our mechanical deformation timescale of $\tau_{m}=10^{-8} \mathrm{~s}$, and therefore support the simulation results of a higher $T_{g}$ value from mechanics compared with volumetric changes.

\subsection{Non-linear Response}

Non-linear mechanics were studied by applying a uniaxial strain on the network structures of composition

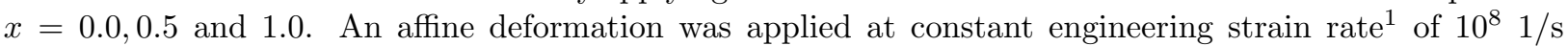
along the axial dimension with constant pressure conditions (anisotropic Nosè-Hoover barostat) in the two lateral dimensions. The network structures were subjected to three independent uni-axial strains along each Cartesian direction in extension and compression. For each composition, three temperatures were considered relative to the $T_{g}[4]: T_{g}-210, T_{g}$, and $T_{g}+210 \mathrm{~K}$. The averaged stress curves for extension and compression are shown in Figure 7. In both extension and compression, all three compositions have the expected stiff response in the glassy state, a weaker response near $T_{g}$, and a soft response in the rubbery state. The stress in both the glassy state and near the glass transition temperature was (1) initially linear, followed by (2) a gradual weakening at $\sim 5 \%$ strain, (3) a sharp yield at $\sim 8 \%$ strain, and finally (4) a near-constant value. The stress in the rubbery regions reflected only one significant change at $\% 5$ strain.

\footnotetext{
${ }^{1}$ The uni-axial strain rate was chosen as the cyclic strain rate averaged over one-fourth cycle at $5 \cdot 10^{10} \mathrm{~Hz}$.
} 


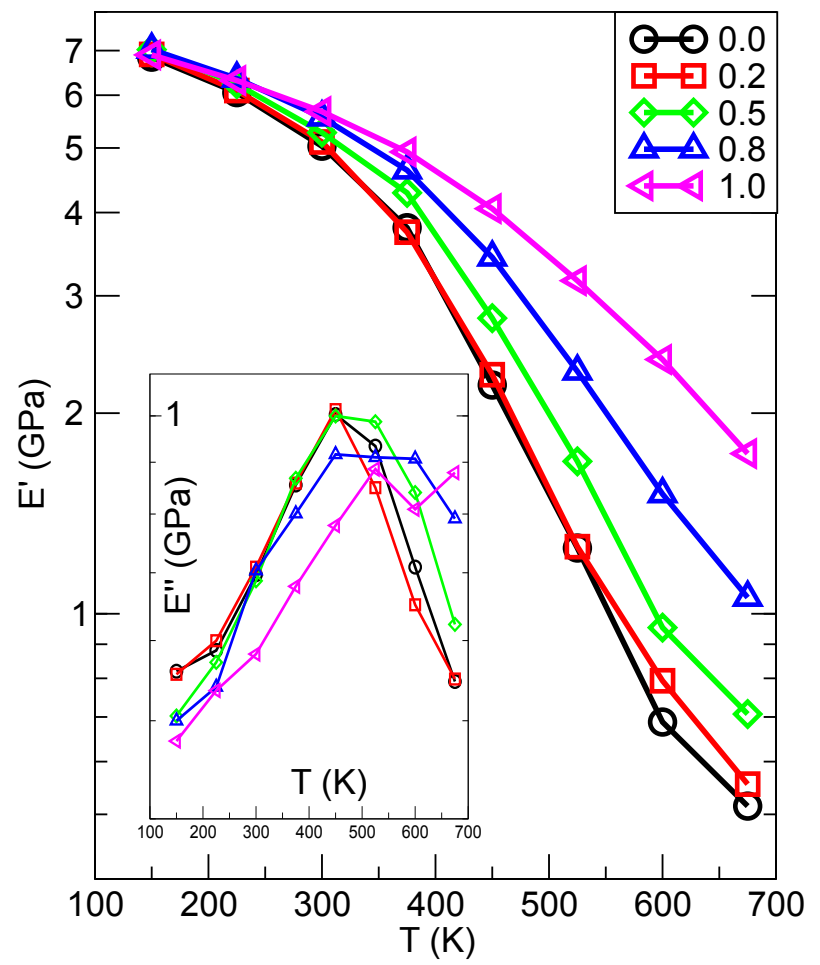

Figure 5. Young's storage modulus $\left(E^{\prime}\right)$ as a function of cross-linker composition $(x)$ and temperature $(T)$. All data corresponds to a cyclic strain with a frequency of $5 \cdot 10^{10} \mathrm{~Hz}$. Inset: Young's loss modulus $\left(E^{\prime \prime}\right)$. The RMS error of fitting the sinusoid waveform ranged from $\sim 0.5$ to $10 \%$ in the glassy and rubbery states, respectively. 


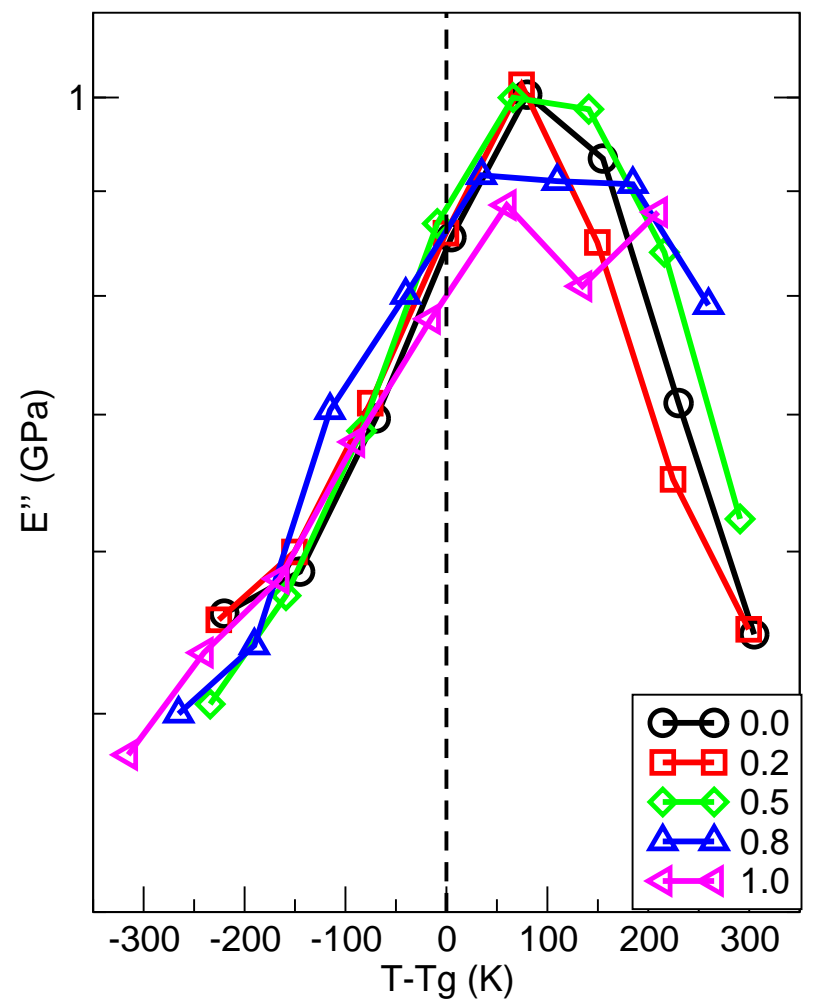

Figure 6. Young's loss modulus $\left(E^{\prime \prime}\right)$ as a function of cross-linker composition $(x)$ and difference from the glass transition temperature $\left(T-T_{g}\right)$, where $T_{g}$ values are taken from the volumetric glass transition in Ref [4]. 
The three compositions displayed similar behavior deep in the glassy state $\left(T=T_{g}-210 \mathrm{~K}\right)$, with only minor differences occurring at high strains. However, for a temperature near $T_{g}$, the composition $x=1.0$ shows higher stresses beyond the yield point. In the rubbery state $\left(T=T_{g}+210 \mathrm{~K}\right)$, the composition $x=1.0$ clearly had the stiffest stress response at all strains while $x=0.0,0.5$ deformed more easily. Compared with $x=1.0$, the relatively softer stress-strain curves of $x=0.5$ above $T_{g}$ suggest that POP units can provide flexibility by bridging the stiffer MCA units of the network.

The glassy modulus can be measured from the tensile deformations described above and compared with the total modulus obtained from the oscillation measurements. We take the modulus at zero strain as $\Delta \sigma / \Delta \epsilon$ where $\Delta \epsilon$ spans the range -0.5 to $0.5 \%$. The values of modulus from uni-axial extension were 6.6 , 6.7 , and $5.9 \mathrm{GPa}$ for compositions $x=0.0,0.5$ and 1.0, respectively. These results agree well with those from the oscillatory deformations presented in Figure 5, which were 6.7, 6.7 and 6.0 GPa, respectively.

Although the network samples considered here are nanoscopic, it is natural to compare the qualitative features of Figure 7 with the experimental data from thermo-mechanical analysis and tensile tests. The simulation results presented here capture the experimental trend of the Young's modulus with composition for both the glassy and rubbery states[2,3], although the absolute modulus and stress values from simulation were substantially higher than experiment[2]. For example, from Figure 7 the simulated yield stress of compositions with purely MCA cross-linkers $(x=1.0)$ was approximately $400 \mathrm{MPa}$ in compression in the glassy state; Quasi-static experiments in the glassy state report a value of $119 \mathrm{MPa}$ [2], which is a factor of 3.3 smaller. At high strains approaching the ultimate strength, nanoscopic mechanical tests of networks become fundamentally different than their macroscopic counterparts due to the influence of defects. Simulations typically do not consider the effect of an interface on properties of the bulk material. This sharply contrasts with tensile experiments, where defects on the sample surface directly affect the ultimate strength. Thus, we consider the stress found from our MD simulations using periodic boundaries as a prediction of an ideal internal mechanical response of a macroscopic tensile test. Further, the high strain rate in simulations can result in an overlap of relaxations in the glassy state and the glass transition, while this is not the case in quasi-static experiments.[5] Lastly, we note that beyond the elastic region, the changes imposed onto the network structure may be accommodated more easily for simulations that allow covalent bonds to be broken.

In both compression and tension, the most striking feature of the stress response is a rapid increase in the range of $0-10 \%$ strain followed by a plateau of approximately constant stress. It is useful to view this feature in terms of the network chain structure. In previous work[4], we identified MCA and POP as relatively stiff and flexible network components, respectively; several chain conformational measures were used to show that, upon heating, the flexible POP chains extended with volumetric (tri-axial) expansion while MCA chains were effectively fixed in length. To study changes in the chain structure, we compute the end-toend distance $(R)$ of the chains (i.e., the distance between crosslinks) during deformation in the glassy state $(T=T g-210 \mathrm{~K})$. Figure 8 shows the changes of the components of end-to-end distance (panel $a-c: R_{\text {lateral }}$, $R_{\text {axial }}$ ) and total change of end-to-end distance (panel $d-f: R$ ) for POP, MCA and DGEBA as computed from the separation of the two terminal heavy atoms in each molecule during compression. As would be expected, the molecules rearrange to accommodate the compression of the simulation box; the component of $R$ for DGEBA, MCA, and POP along the axial direction of the strain decreases, while the components perpendicular to the deformation direction are increased. At small strain, the magnitude of $R$ is preserved. However, $R$ begins to increase at $\sim 5 \%$ strain for POP and at $\sim 10 \%$ strain for MCA, DGEBA $(x=0.0)$, and DGEBA $(x=1.0)$. These values of strain approximately correspond to the yield point in the glassy state for the two compositions shown in Figures 7 and 8, suggesting that the networks can accommodate uni-axial deformation up to $\sim 10 \%$ without substantial changes to the intra-chain configurations. Beyond $10 \%$ strain, the cross-links have moved far enough apart in the lateral directions that DGEBA molecules begin to stretch and POP chains have began to uncoil; these mechanisms coincide with the onset of a post-yield plateau stress.

In each of the stress-strain curves shown in Figure 7, the stresses increase with MCA composition at large strain $(\sim 15 \%)$. This can be rationalized by recognizing that stiffness is provided by both the high crosslink density of MCA networks and, as we have discussed previously[4], the rigidity of the MCA molecule which does not allow a meaningful amount of chain bending. Chain extension may be less prevalent in experimental tensile tests, since tightly-crosslinked glassy networks fail by brittle fracture in experiments at 


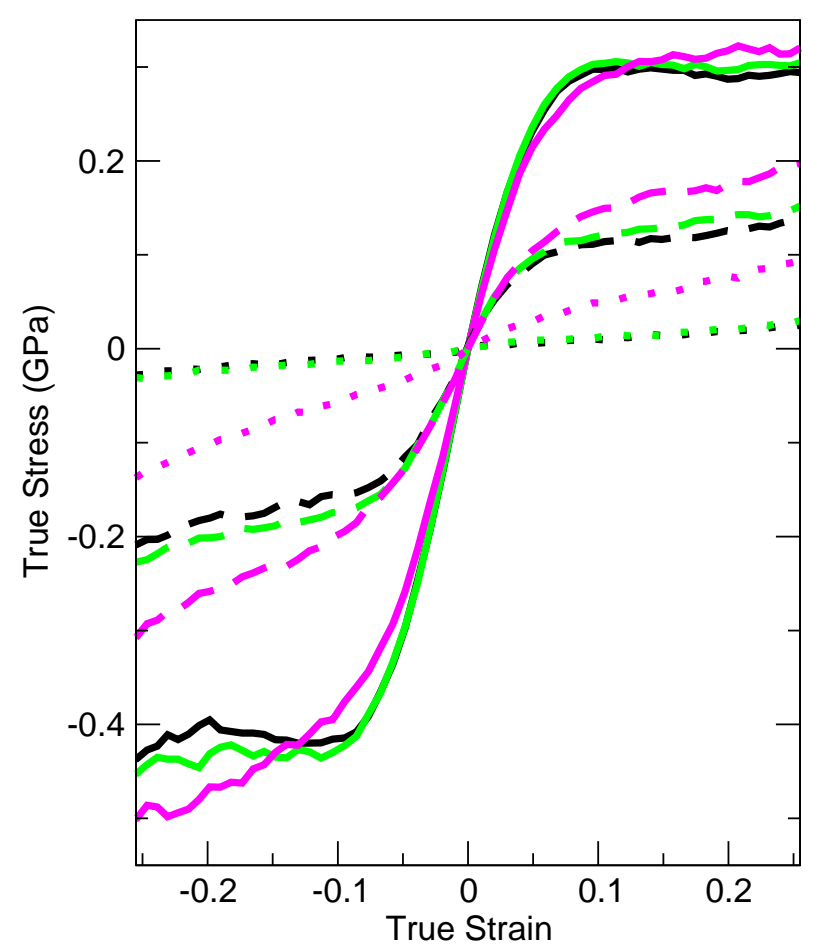

Figure 7. Stress-strain curves as a function of composition $(x)$ for temperatures $T=T_{g}-210$ (solid), $T_{g}$ (dash), and $T_{g}+$ $210 \mathrm{~K}$ (dot). Results are shown for the compositions $x=0.0$ (black), $x=0.5$ (green), and $x=1.0$ (magneta). Each curve is a composite of stress data taken from a compression $(\epsilon<0)$ and extension strain $(\epsilon>0)$ at a strain rate of $10^{8} 1 / \mathrm{s}$.

strains in the range of $3 \%-10 \%$. Although it is not yet clear how the strain observed on the macroscopic level is locally distributed, previous nano-scale molecular simulations have suggested glassy epoxies are capable of supporting strains of approximately $20 \%$ before failure of the matrix.[30]

\section{Conclusions}

The mechanical properties of thermoset epoxy networks were studied at high strain rates using atomistic molecular dynamics simulations. Five bi-modal epoxy networks were considered by varying the composition of amine crosslinkers between the long, highly flexible POP $(x=0.0)$ and the short, rigid MCA $(x=1.0)$. Simulations of cyclic deformation were used to characterize the viscoelastic behavior of each composition. We first estimated the maximum frequency corresponding to a linear viscoelastic regime by considering the thermal response, energy dissipation, speed of pressure waves and magnitude of the inertial forces. Similar to experimental dynamic modulus measurements, we observed a well-defined reduction in the storage modulus with temperature, and a peak of the loss modulus. The breadth of the glass transition of the storage modulus increased with MCA content and, in all cases, the glass transition region was found to be much broader than in experiments at low strain-rates.

We have previously reported that the volumetric and enthalpic changes during the cooling predict the $T_{g}$ to be well-above the experimental values; this difference was understood to be due to the high cooling rates employed in molecular dynamics simulation, which cause the system to fall out of equilibrium at a higher temperature.[5, 4] In addition to the upward shift of the volumetric glass transition temperature relative to experiments, the mechanical glass transition measured from cyclic deformation simulations was even higher by approximately $100 \mathrm{~K}$ or more, depending on the material composition. The difference of the two values was attributed to the relatively shorter timescales of mechanical deformation compared to that of the volume changes in cooling simulations. We found the molecular structures at temperatures above 

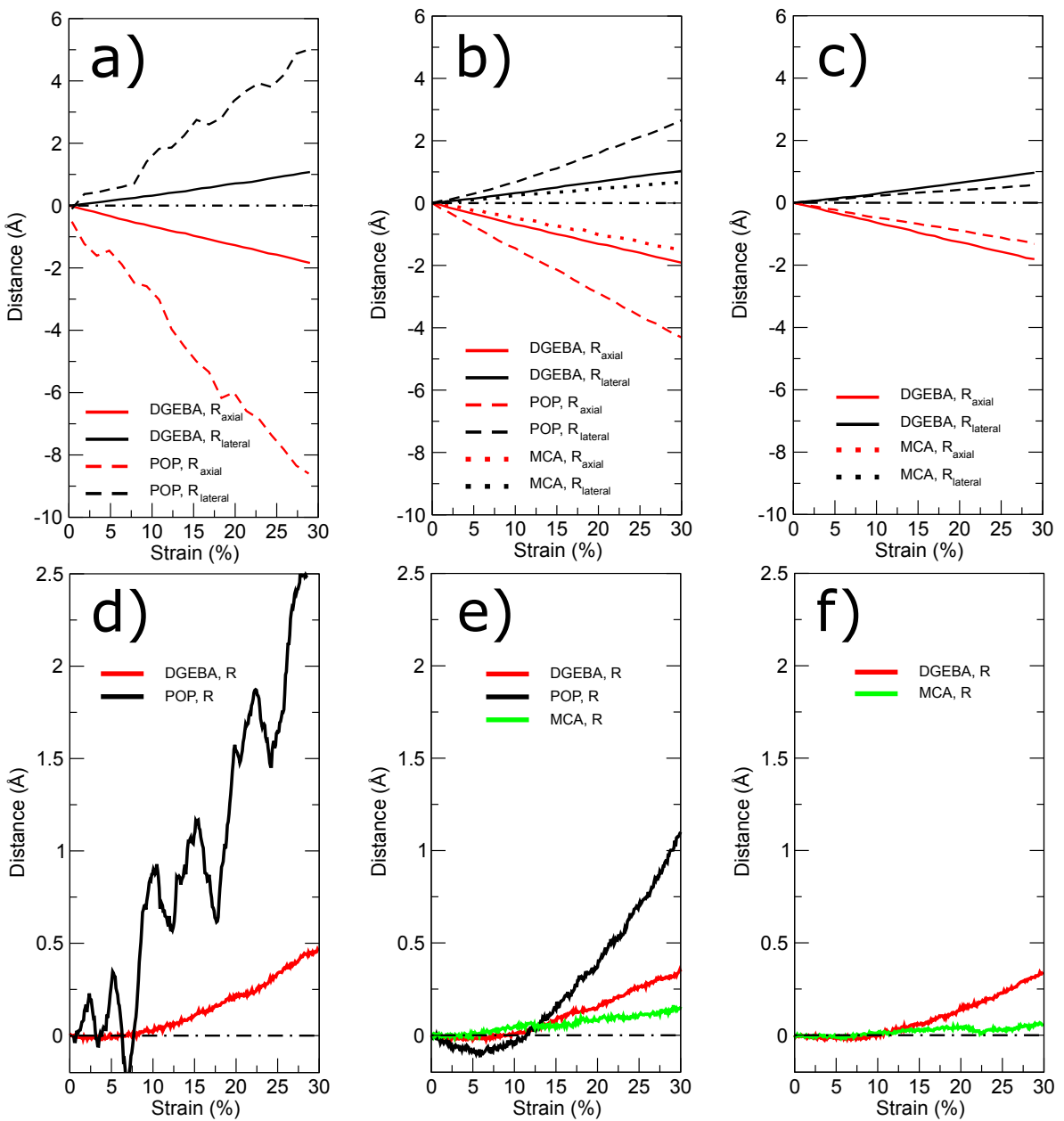

Figure 8. The change of the end-to-end distance $R$ of DGEBA, POP, and MCA molecules during compression in the glassy state. (a-c): Axial and lateral changes of $R$ for compositions $x=0.0,0.5$, and 1.0, respectively, during strain. (d-f): Total change of $R$ during strain. The zero value is marked by a dot-dash line.

the volumetric glass transition to be well-suited for studying viscoelasticity, since these structures are at equilibrium and not sensitive to high cooling rates. Further, the breadth of the glass transition and value of $T_{g}$ were shown to be related through the shape of the temperature-dependent relaxation time, and the broad glass transitions seen in our simulations were attributed to this relationship.

The mechanical response beyond the elastic limit was studied from uni-axial strains at temperatures below, near, and above the glass transition in both compression and tension. The networks had a similar stress response in the glassy state and at low strains. At higher temperatures and higher strains, the MCA-containing networks $(x>0.0)$ exhibited a stiffer response than the POP network $(x=0.0)$; this stiffness was attributed to the increased cross-link density and rigidity of the MCA molecule. For all of the network compositions, the stress response in both the glassy state and near the glass transition temperature was initially linear, followed by a yield and plateau behavior. The onset of this behavior occurred at approximately the same strain as the increase of the end-to-end distance of POP and DGEBA molecules. Although the MCA molecule was rigid and did not have any meaningful extension at high strains, the DGEBA molecules in the DGEBA:MCA network $(x=1.0)$ deformed substantially, as measured by the change of the end-to-end distance. In DGEBA:POP networks $(x=0.0)$, the end-to-end distance of both the DGEBA and POP molecules increased. These results suggest the stretching of the network chains as 
a mechanism of a well-defined plateau stress at strains of $10 \%$ to $30 \%$. Overall, our results show that the flexibility of the cross-linker has a significant effect on the linear and nonlinear mechanics, the character of the glass transition of the modulus, and the chain-level structure during strain.

\section{Acknowledgments}

The work of MK and RK was supported by U.S. Army/Battelle Memorial Institute contract number US0010000287704.

\section{References}

[1] P. M. Cunniff, Proceedings of the 18th International Symposium of Ballistics, 1999, pp. 1303-1310.

[2] D. B. Knorr, J. H. Yu, A. D. Richardson, M. D. Hindenland, I. M. McAninch, J. L. Scala and J. L. Lenhart, Polymer, 2012, 53, 5917-5923

[3] K. A. Masser, D. B. Knorr, M. D. Hindenlang, H. Y. Jian, A. D. Richardson, K. E. Strawhecker, F. L. Beyer and J. L. Lenhart, Polymer, 2015, 58, 96-106.

[4] T. W. Sirk, M. Karim, K. S. Khare, J. L. Lenhart, J. W. Andzelm and R. Khare, Polymer, 2015, 58, $199-208$.

[5] T. Sirk, K. Khare, M. Kiram, J. Lenhart, J. Andzelm, G. McKenna and R. Khare, Polymer, $2013,54,7048-7057$.

[6] P. H. Lin and R. Khare, Macromolecules, 2009, 42, 4319-4327.

[7] J. Wang, R. M. Wolf, J. W. Caldwell, P. A. Kollman and D. A. Case, J Comp Chem, 2004, 25, 1157-1174.

[8] J. Wang, W. Wang, P. A. Kollman and D. A. Case, J Mol Graphics Modell, 2006, 25, $247-260$.

[9] H. Sun, J Phys Chem B, 1998, 102, 7338-7364.

[10] A. Jakalian, B. L. Bush, D. B. Jack and C. I. Bayly, J Comput Chem, 2000, 21, 132-146.

[11] A. Jakalian, D. B. Jack and C. I. Bayly, J Comput Chem, 2002, 23, 1623-1641.

[12] R. W. Hockney and J. W. Eastwood, Computer Simulation using Particles, Institue of Physics Publishing, 1988.

[13] S. J. Plimpton, J. Comput. Phys., 1995, 117, 1-19.

[14] Shinoda, Shiga and Mikami, Phys Rev B, 2004, 69, 134103.

[15] T. Sirk, S. Moore and E. Brown, J. Chem. Phys., 2013, 138, 064505.

[16] F. Khabaz, K. S. Khare and R. Khare, TIMES OF POLYMERS (TOP) AND COMPOSITES 2014: Proceedings of the 7th International Conference on Times of Polymers (TOP) and Composites, 2014, pp. 262-265.

[17] K. Yashiro, M. Naito, Y. Minagawa and Y. Tomita, Transaction of the Japan Society of Mechanical Engineers, 2006, 72, $277-284$.

[18] K. Yashiro, Y. Koga, M. Naito, Y. Minagawa and Y. Tomita, Transaction of the Japan Society of Mechanical Engineers, 2009, 75, 881-888.

[19] K. Yashiro, M. Naito, S. Ueno and F. Jie, J Mech Sci, 2010, 52, 136-145.

[20] C. Li, E. Jaramillo and A. Strachan, Polymer, 2013, 54, 881-890.

[21] R. Ewoldt, P. Winter, J. Maxey and G. McKinley, Rheol Acta, 2010, 49, 191-212.

[22] B. Arman, Q. An, S. Luo, T. Desai, D. Tonks, T. Çă̆ın and W. Goddard Iii, Journal of Applied Physics, 2011, 109, 013503.

[23] D. N. Theodorou and U. Suter, Macromolecules, 1986, 19, 379-387.

[24] R. Matheson, Macromolecules, 1987, 20, 1847-1851.

[25] P. in 't Veld and G. Rutledge, Macromolecules, 2003, 36, 7358-7365.

[26] S. Yang and J. Qu, Polymer, 2012, 53, 4806-4817.

[27] L. McGrath, R. Parnas, S. King, J. Schroeder, D. Fischer and J. Lenhart, Polymer, 2008, 49, $999-1014$.

[28] I. Mcaninch, G. Palmese, J. Lenhart and J. L. Scala, Journal of Applied Polymer Science, 2013, 130, $1621-1631$.

[29] J. Schmelzer and T. Tropin, J Chem Phys, 2013, 138, 034507.

[30] G. M. Odegard, B. D. Jensen, S. Gowtham, J. Wu, J. He and Z. Zhang, Chemical Physics Letters, 2014, 591, $175-178$. 